\title{
Significance of hypoxia-inducible factor-1 $\alpha$ expression with atrial fibrosis in rats induced with isoproterenol
}

\author{
FANGJU SU, WEIZE ZHANG, YONGQING CHEN, LING MA, HANPING ZHANG and FEI WANG
}

Department of Cardiology, Lanzhou General Hospital, People's Liberation Army, Lanzhou, Gansu 730050, P.R. China

Received February 24, 2014; Accepted August 20, 2014

DOI: 10.3892/etm.2014.1989

\begin{abstract}
Atrial interstitial fibrosis plays a dual role in inducing and maintaining atrial fibrillation (AF). Hypoxia-inducible factor-1 $\alpha(\mathrm{HIF}-1 \alpha)$ has been reported as closely associated with renal, liver and pulmonary fibrosis diseases. However, whether HIF- $1 \alpha$ is involved in myocardial fibrosis, and the associations between HIF- $1 \alpha$, transforming growth factor- $\beta_{1}$ (TGF- $\beta_{1}$ ) and matrix metalloproteinase-9 (MMP-9) remain unknown. Therefore, this area warrants studying for the significance of AF diagnosis and treatment. The present study investigated the expression of HIF-1 $\alpha$ in atrial fibrosis and its possible mechanism in isoproterenol (ISO)-induced rats. The three groups of rats; control, ISO and ISO plus sirolimus [also known as rapamycin (Rapa)], were treated for 15 days and sacrificed to remove the myocardial tissues. The expression levels of HIF- $1 \alpha$, TGF- $\beta_{1}$ and MMP-9 and their associations with atrial fibrosis were examined through histomorphology and protein and mRNA levels. The protein and mRNA levels of HIF- $1 \alpha$, TGF- $\beta_{1}$ and MMP-9 in the ISO group were increased markedly $(\mathrm{P}<0.01)$ compared with the control group, while those in the Rapa group were clearly decreased $(\mathrm{P}<0.01)$ compared with the ISO group. The protein and mRNA levels of HIF- $1 \alpha$, TGF- $\beta_{1}$ and MMP-9 were positively correlated $(\mathrm{P}<0.01)$ with atrial fibrosis (collagen volume fraction index), as were the $H I F-1 \alpha, T G F-\beta_{1}$ and $M M P-9$ mRNA levels $(\mathrm{P}<0.01)$ and the mRNA levels between MMP-9 and TGF- $\beta_{I}$ $(\mathrm{P}<0.01)$. During the process of atrial fibrosis in ISO-induced rats, HIF- $1 \alpha$ promotes the expression of TGF- $\beta_{1}$ and MMP-9 protein, and thus is involved in in atrial fibrosis.
\end{abstract}

\section{Introduction}

Clinically, atrial fibrillation (AF), which is of one of the most common types of arrhythmia, shows high disability

Correspondence to: Professor Weize Zhang, Department of Cardiology, Lanzhou General Hospital, People's Liberation Army, 333 Southern Binhe Road, Lanzhou, Gansu 730050, P.R. China

E-mail: zhangzwz123@126.com

Key words: hypoxia inducible factor- $1 \alpha$, atrial, fibrosis, transforming growth factor- $\beta_{1}$, matrix metalloproteinase- 9 and mortality rates in patients $(1,2)$. In recent years, angiotensin II (AngII) and AF occurrence and maintenance has experienced increasing attention. The AngII levels in AF increase and eventually induce atrial fibrosis (3). Atrial fibrosis plays dual roles in inducing and maintaining AF (4-6). A previous study (7) showed that the expression level of hypoxia-inducible factor- $1 \alpha(\mathrm{HIF}-1 \alpha)$ is associated with AngII, which is involved in renal fibrosis. However, no associated study has been conducted for myocardial fibrosis. The present study refers to the method by Zhang et al (8), which used a subcutaneous bolus injection of isoproterenol (ISO) to induce AngII expression to establish an atrial fibrosis rat model. The HIF-1 $\alpha$ inhibitor (9) [sirolimus, also known as rapamycin (Rapa)] was administered to examine the protein expression and mRNA levels of AngII, HIF-1 $\alpha$, transforming growth factor-beta ${ }_{1}\left(\mathrm{TGF}-\beta_{1}\right)$ and matrix metalloproteinase-9 (MMP-9) in myocardial tissue in the atrial fibrosis rat model, and thus the present study investigated their relevance and the possible mechanism of how HIF-1 $\alpha$ following the ISO injection would induce atrial fibrosis.

\section{Materials and methods}

Animal model. Thirty healthy male Wistar rats, $180 \pm 20 \mathrm{~g}$ body weight, were purchased from the Lanzhou School of Medicine Animal Center in Lanzhou University (Lanzhou, China), and were maintained at $20-25^{\circ} \mathrm{C}$ with lighting-controlled circadian rhythms (8:00 am-10:00 pm) under normal feeding with free food and water. The rats were randomly divided into three groups of 10 rats: Control, ISO and ISO plus sirolimus (Rapa). The animal experiment was approved by the Animal Ethics Committee. The study referred to the method by Zhang et al (8) to establish an atrial fibrosis rat model. The ISO group rats were administered multipoint subcutaneous bolus injections of hydrochloric acid ISO (batch no. 080705; Shanghai Hefeng Pharmaceutical Co., Ltd., Shanghai, China), $5 \mathrm{mg} / \mathrm{g} / \mathrm{day}$, and once per day for seven days. The Rapa-intervention group rats were provided sirolimus oral solution (batch no. 110901; Hangzhou Zhongmei Huadong Pharmaceutical Co., Ltd., Hangzhou, China), specification $50 \mathrm{ml}: 50 \mathrm{mg}$, initiated on the second of the same ISO treatment as in the ISO group, $3 \mathrm{mg} / \mathrm{kg} /$ day (10), once per day and gavage for 14 days, with the interval between gavage and subcutaneous injection being 4-6 h. Simultaneously, the control and ISO groups were separately administered an equal amount of double-distilled water 
for stomach gavage as for the Rapa group. All the rats were sacrificed by cervical dislocation after 15 days.

Sample collection and preservation. Along the coronary plane maximum transverse diameter, partial myocardial tissue was cut and placed in $10 \%$ formaldehyde solution for $24 \mathrm{~h}$ fixation. Following this, the tissue was paraffin-embedded and five serial slices $(4-\mu \mathrm{m})$ were cut from it. Two slices were used for hematoxylin and eosin (HE) and Masson staining to observe the extent of myocardial fibrosis, which used collagen volume fraction (CVF) as the atrial fibrosis index. Immunohistochemistry (IHC) was performed on the remaining three slices to detect the expression of HIF- $1 \alpha$, TGF- $\beta_{1}$ and MMP-9. The samples were obtained from the remaining cardiac tissue for detection of AngII by radioimmunoassay, and HIF- $1 \alpha$, TGF- $\beta_{1}$ and MMP-9 expression levels by western blot (WB) analysis and reverse transcription quantitative polymerase chain reaction (RT-qPCR). The remaining cardiac tissue was cryopreserved in liquid nitrogen.

Detection of AngII level in the myocardium in rats. A radioimmunoassay kit (Beijing North Institute of Biotechnology, Beijing, China) was used to detect the concentration of AngII.

Observation of myocardial fibrosis. Myocardial tissue underwent formaldehyde fixation, dehydration, transparency, embedding in paraffin and slicing into two $4-\mu \mathrm{m}$ sections. HE and Masson staining were subsequently performed, and the sections were mounted and observed by light microscope and radiography.

The CVF $[\mathrm{CVF}=$ (collagen area/total area of view field) $\mathrm{x} 100 \%$ ] calculation was as follows: Three non-vascular vision images (magnification, x400) were selected from each Masson staining slice. The image scanning software, Image-Pro Plus 6.0 (Media Cybernetics, Inc., Rockville, MD, USA), was used for image analysis and myocardial CVF calculation.

IHC. Myocardial tissue paraffin sections underwent a number of steps, including dewaxing, antigen hot fix, blocking solution incubation, first and secondary antibody incubation, diaminobenzidine coloration, counterstaining, transparency and mounting. Phosphate-buffered saline (PBS) was used to replace the first antibody as the negative control, and the positive control was provided in the IHC kit (Boster Biological Tech Ltd., Wuhan, China). Rabbit anti-rat antibodies of HIF-1 $\alpha$, TGF- $\beta_{1}$ and MMP-9 were all purchased from Santa Cruz Biotechnology, Inc. (Santa Cruz, CA, USA). The first antibody was diluted by 1:50, and the secondary goat anti-rabbit antibody was provided by Jackson ImmunoResearch Laboratories, Inc. (West Grove, PA, USA) under the dilution of 1:500.

WB analysis. Myocardial tissue was placed in liquid nitrogen pre-cooling mortars, and a proper amount of protein lysate was added and centrifuged. Subsequent to removal of the supernatant, a small portion of the precipitation was used for the determination of protein concentration. Protein $(100 \mu \mathrm{g})$ was mixed with $5 \mathrm{X}$ protein electrophoresis loading buffer, placed into a boiling water bath for $5 \mathrm{~min}$, centrifuged at $12,000 \mathrm{x}$ g for $1 \mathrm{~min}$ and fully loaded along with the protein marker (Fermentas, Waltham, MA, USA). In Tris-glycine buffer ( $\mathrm{pH}$ 8.0) and under an $80 \mathrm{~V}$ voltage, a 1.5-2 h electrophoresis was performed, followed by a $20 \mathrm{~V}$ constant voltage and $1.5 \mathrm{~h}$ transferring to nitrocellulose film (Millipore, Billerica, MA, USA). The film was removed and $5 \%$ skimmed milk powder-sealed liquid/PBS with Tween (PBST) was added under room temperature and slow agitation was performed for $1.5 \mathrm{~h}$. First antibody incubation was as follows: HIF- $1 \alpha$, TGF- $\beta_{1}$ and MMP-9 antibodies were purchased from Santa Cruz Biotechnology, Inc., and diluted 1:300 in 5\% skimmed milk powder/PBST at $4^{\circ} \mathrm{C}$ overnight; and anti-GAPDH (Santa Cruz Biotechnology, Inc.) monoclonal antibody was 1:10,000 diluted in 5\% skimmed milk powder/PBST at $4^{\circ} \mathrm{C}$ overnight. PBST was used to wash the membrane three times for $10 \mathrm{~min}$ each. Secondary antibody incubation was performed as follows: Goat anti-rabbit antibody was 1:2,000 diluted in 5\% skimmed milk powder/PBST and sheep anti-mouse (second for GAPDH) was 1:2,000 diluted in $5 \%$ skimmed milk powder/PBST, incubated at room temperature for $1 \mathrm{~h}$, followed by washing of the membrane three times with PBST for 15 min each. The film was placed in the SuperSignal ${ }^{\mathrm{TM}}$ West Pico (Pierce, Rockford, IL, USA) for $2 \mathrm{~min}$, tableted and developed to detect specific protein bands. The gel imaging system was photographed and the strip area and gray analysis of the protein zone was expressed by the integral gray value (D).

$R T$ - $q P C R$. TRIzol ${ }^{\circledR}$ was added to $0.1 \mathrm{~g}$ myocardial tissue to extract RNA; 1-4 $\mu \mathrm{g}$ RNA was obtained for RT-qPCR. CFX-96 (Bio-Rad, Hercules, CA, USA) was used for fluorescence qPCR and the $20 \mu \mathrm{l}$ PCR amplification reaction system was performed. Primer sequences were as follows: HIF-l $\alpha$ forward, 5'-ATCTCGGCGAAGCAAAGAGT-3'; reverse, 5'-TGACCA TCATCTGTTAGCACCAT-3'; $T G F-\beta_{1}$ forward, 5'-CTAATG GTGGACCGCAACAAC-3'; reverse, 5'-TAACGCCAGGAA TTGTTGCTAT-3'; $M M P-9$ forward, 5'-CAAACCCTGCGT ATTTCCATT-3', reverse, 5'-ACATCTCTCCTGCCGAGT TGC-3'; and GAPDH forward, 5'-AGTGCCAGCCTCGTC TCATAG-3', reverse, 5'-CGTTGAACTTGC CGTGGGTAG-3'. GAPDH was used as the internal control. Each sample was loaded in three replicates. HIF-1 $\alpha, T G F-\beta_{1}, M M P-9$ and $G A P D H$ RT-qPCR amplification conditions were as follows: Annealing temperature at $95^{\circ} \mathrm{C}$ for $15 \mathrm{sec}$, extension temperature at $60^{\circ} \mathrm{C}$ for $20 \mathrm{sec}$ and melting curve temperature at $72^{\circ} \mathrm{C}$ for $20 \mathrm{sec}$, in a total of 45 cycles. At the end of the reaction, fluorescence quantitative data were collected including the amplification curve, working curve, melting curve and the corresponding $\mathrm{Ct}$ value, according to the $2^{-\triangle \mathrm{mRNA} \Delta \mathrm{Ct}}$ method to determine the relative mRNA expression level.

Statistical methods. SPSS 17.0 software (SPSS, Inc., Chicago, IL, USA) was used for statistical analysis. All data are shown as mean \pm standard deviation. Single factor analysis of variance was used for inter-group comparison, least significant difference method was used for pairwise comparison, and the Pearson method was used for product-moment correlation analysis. $\mathrm{P}<0.05$ was considered to indicate a statistically significant difference. 
Table I. Western blot analysis and RT-qPCR results of HIF- $1 \alpha$, TGF- $\beta_{1}$ and MMP-9.

\begin{tabular}{lcccccccc}
\hline & \multicolumn{2}{c}{ HIF-1 $\alpha$} & & \multicolumn{2}{c}{ TGF- $\beta_{1}$} & & \multicolumn{2}{c}{ MMP-9 } \\
\cline { 2 - 3 } \cline { 7 - 8 } Group & Protein & mRNA & & Protein & mRNA & & Protein & mRNA \\
\hline Control & $0.294 \pm 0.021$ & $(5.795 \pm 0.822) \times 10^{-5}$ & & $0.660 \pm 0.059$ & $(1.223 \pm 0.085) \times 10^{-2}$ & & $0.974 \pm 0.116$ & $(2.596 \pm 0.193) \times 10^{-6}$ \\
ISO & $0.522 \pm 0.039^{\mathrm{a}}$ & $(2.103 \pm 0.492) \times 10^{-4 \mathrm{a}}$ & & $1.444 \pm 0.081^{\mathrm{a}}$ & $(7.511 \pm 0.156) \times 10^{-2 \mathrm{a}}$ & & $1.939 \pm 0.113^{\mathrm{a}}$ & $(4.854 \pm 0.210) \times 10^{-6 \mathrm{a}}$ \\
Rapa & $0.279 \pm 0.016^{\mathrm{b}}$ & $(6.374 \pm 0.993) \times 10^{-5 \mathrm{~b}}$ & & $0.669 \pm 0.040^{\mathrm{b}}$ & $(1.205 \pm 0.090) \times 10^{-2 \mathrm{~b}}$ & & $0.916 \pm 0.125^{\mathrm{b}}$ & $(2.789 \pm 0.180) \times 10^{-6 \mathrm{~b}}$ \\
\hline
\end{tabular}

${ }^{a} \mathrm{P}<0.01$ compared with the control group; ${ }^{\text {}} \mathrm{P}<0.01$ compared with the ISO group. RT-qPCR, reverse transcription quantitative polymerase chain reaction; ISO, isoproterenol; Rapa, ISO plus sirolimus; HIF- $1 \alpha$, hypoxia-inducible factor- $1 \alpha$; TGF- $\beta 1$, transforming growth factor- $\beta 1$; MMP-9, matrix metalloproteinase-9.

control group
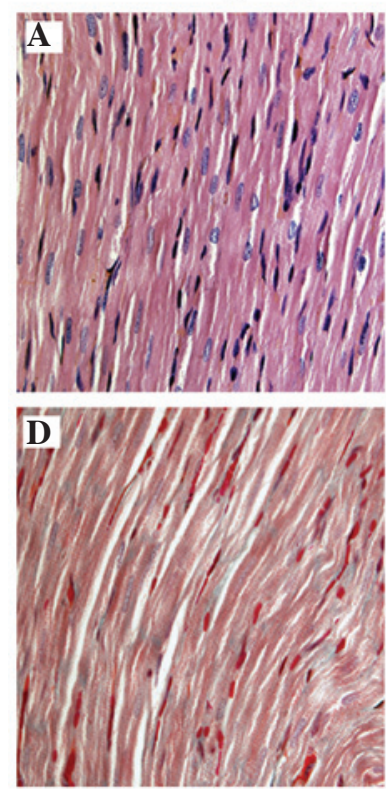

IS0 group
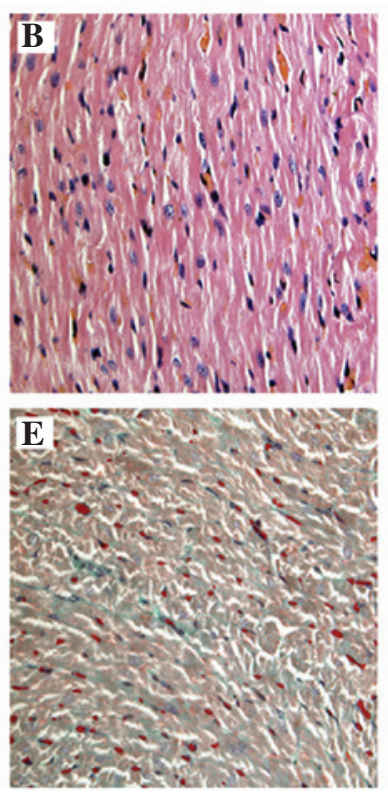

Rapa group
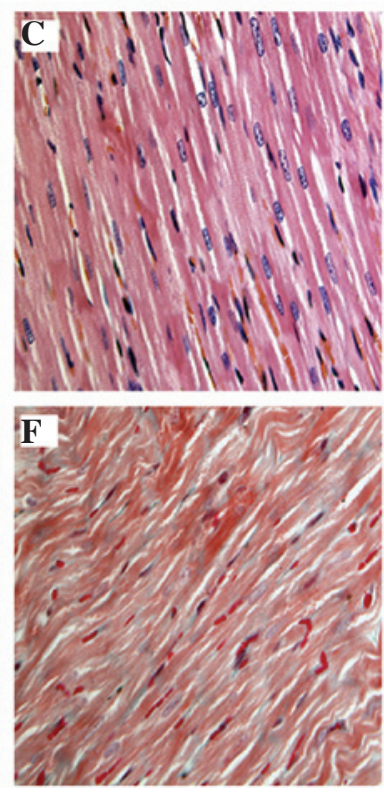

Figure 1. Myocardial interstitial fibrosis performance. (A-C) Hematoxylin and eosin staining; (D-F) Masson staining (green represents the myocardial interstitial collagen component). (A and D) Control; (B and E) ISO; and (C and F) Rapa groups (magnification, x400). ISO, isoproterenol; Rapa, ISO plus sirolimus.

\section{Results}

Myocardial interstitial fibrosis. HE staining showed that the control group exhibited normal space between the myocardial nuclei, regular shape of the nuclei along the heart muscle and structured alignment of myocardial interstitial fibrosis (Fig. 1A). The ISO group had increased myocardial interstitial components and widened nuclear space. Fibrosis and cardiac muscle fiber shapes were disordered (Fig. 1B). The Rapa group showed a degree of reduction compared with the ISO group in the shape of the nuclei, and myocardial interstitial fibrous and interstitial fiber arrangements were disordered (Fig. 1C).

The Masson staining and CVF results showed that under light microscopy, normal myocardial interstitial collagen components appeared green (light green counterstained), the nuclei appeared blue, and myocardial fibers, cytoplasm and red blood cells appeared red (Fig. 1D-F). The image was analyzed to calculate the level of atrial fibrosis (CVF index), taking the average value as the measurement value. The control group
$(15.482 \pm 0.837 \%)$ did not show atrial fibrosis, and the Rapa group (16.730 $\pm 1.052 \%)$ showed a greatly reduced atrial fibrosis level compared with the ISO group $(86.704 \pm 1.982 \%)(\mathrm{P}<0.01)$. The difference between the Rapa and control groups did not show a statistically significant difference $(\mathrm{P}>0.05)$.

Ang II levels in the myocardium of rats by radioimmunoassay. The result showed that the ISO $(139.402 \pm 4.431 \mathrm{ng} / \mathrm{l})$ and Rapa $(132.712 \pm 5.316 \mathrm{ng} / \mathrm{l})$ groups had significantly increased Ang II levels $(\mathrm{P}<0.01)$ compared with the control group $(31.172 \pm 7.271 \mathrm{ng} / \mathrm{l})$.

IHC. Immunohistochemical detection of HIF- $1 \alpha$, TGF- $\beta_{1}$ and MMP-9 expression showed claybank color for positive staining in myocardial cells of the rats under microscopy, distributed throughout the myocardial cytoplasm. The ISO group showed stronger expression levels than the control group, while the Rapa group showed markedly reduced levels of expression compared with the ISO group (Fig. 2). 

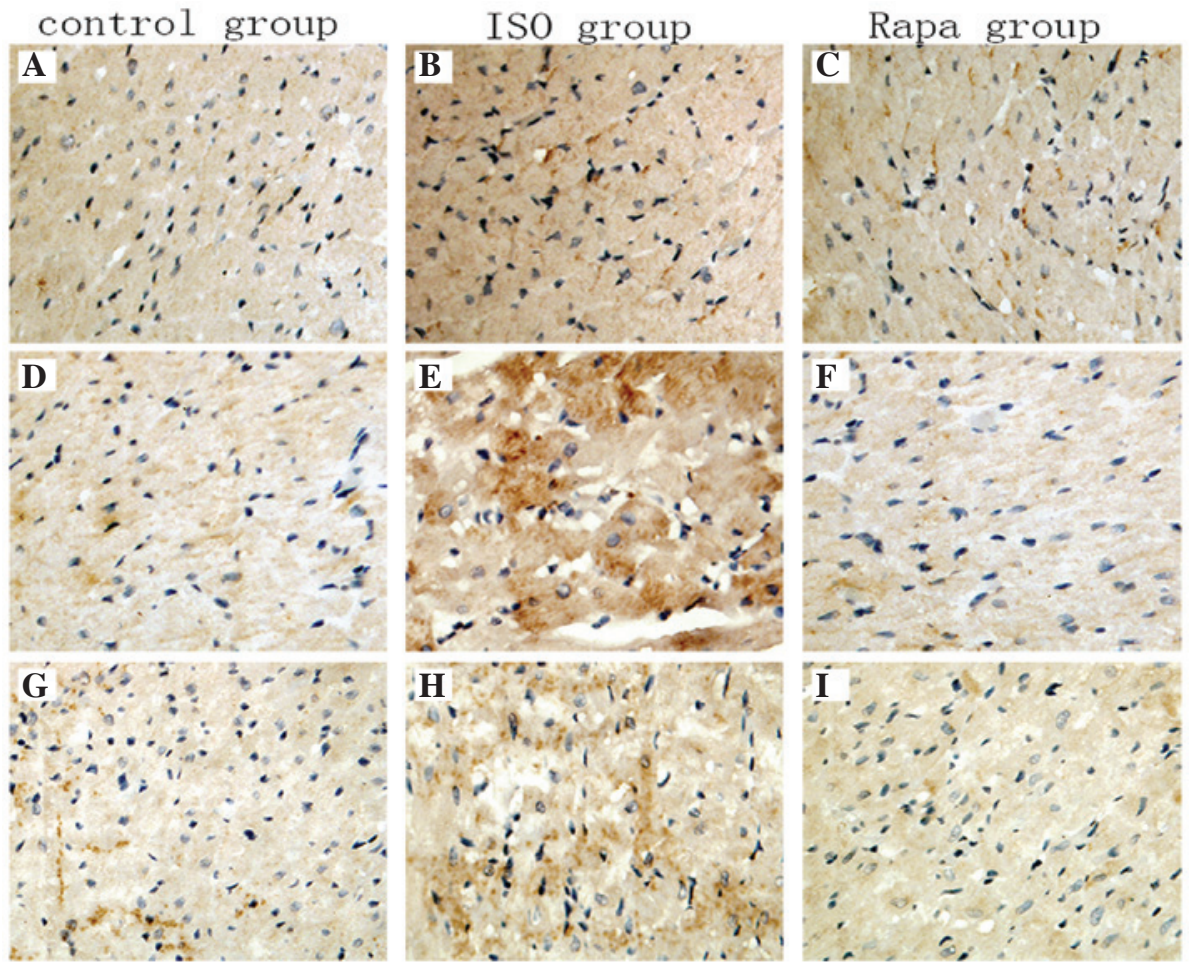

Figure 2. Immunohistochemistry results of HIF-1 $\alpha$, TGF- $\beta_{1}$ and MMP-9 expression. Comparison of HIF-1 $\alpha$, TGF- $\beta_{1}$ and MMP-9 expression in myocardial cells (claybank spots) in the cytoplasm represents positive staining. (A, D and G) Control; (B, E and H) ISO; and (C, F and I) Rapa groups (magnification, x400). ISO, isoproterenol; Rapa, ISO plus sirolimus; HIF-1 $\alpha$, hypoxia-inducible factor- $1 \alpha$; TGF- $\beta_{1}$, transforming growth factor- $\beta_{1}$; MMP-9, matrix metalloproteinase-9.
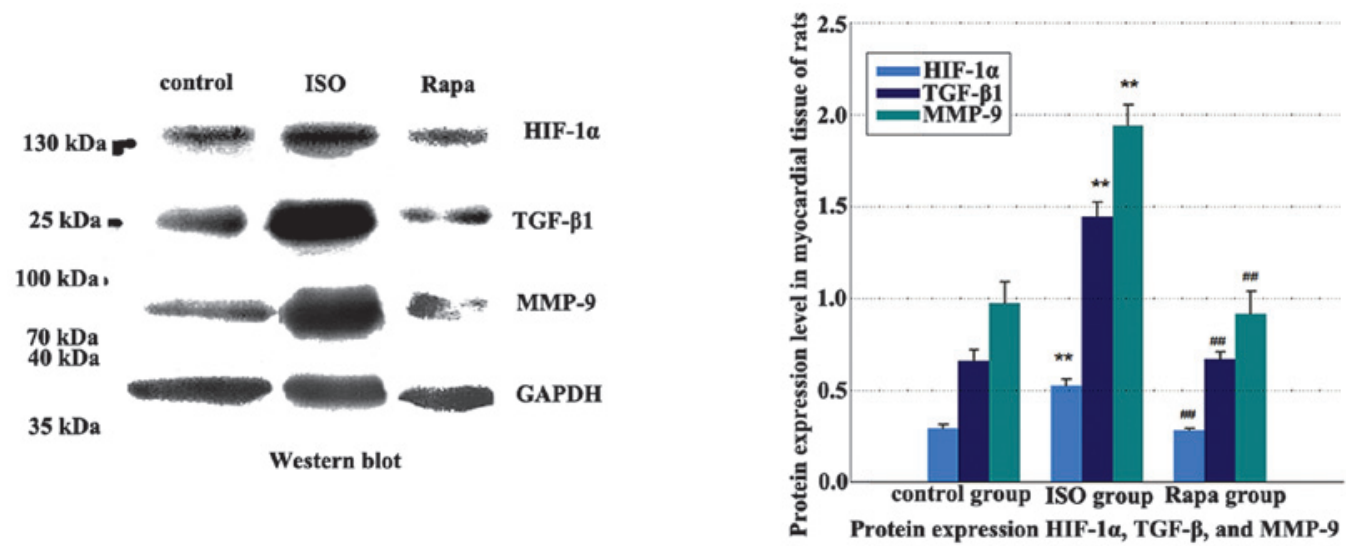

Figure 3. Protein expression of HIF-1 $\alpha$, TGF- $\beta_{1}$ and MMP-9. ${ }^{* *} \mathrm{P}<0.01$ compared with the control group, ${ }^{\# \#} \mathrm{P}<0.01$ compared with the ISO group. ISO, isoproterenol; Rapa, ISO plus sirolimus; HIF-1 $\alpha$, hypoxia-inducible factor-1 $\alpha$; TGF- $\beta_{1}$, transforming growth factor- $\beta_{1}$; MMP-9, matrix metalloproteinase-9.

WB analysis and RT- $q P C R$. The HIF-1 $\alpha, T G F-\beta_{l}$ and MMP-9 at the mRNA and protein levels were higher in the ISO group than those in the control group. The mRNA and protein expression in the Rapa group were significantly lower than those in the ISO group (Table I and Fig. 3).

Associations among HIF-1 $\alpha, T G F-\beta_{1}$ and MMP-9 expression levels in muscle fibrosis. In myocardial tissue, $H I F-1 \alpha, T G F-\beta_{l}$ and MMP-9 mRNA expression levels were positively correlated $(\mathrm{r}=0.919,0.997$ and $0.985 ; \mathrm{P}<0.01)$ with atrial fibrosis (CVF index). HIF-1 $\alpha, T G F-\beta_{1}$ and MMP-9 mRNA expression levels themselves also exhibited a significant positive correlation $(\mathrm{r}=0.936$ and $0.888 ; \mathrm{P}<0.01) . M M P-9$ and $T G F-\beta_{l}$ mRNA expression levels were positively correlated $(\mathrm{r}=0.981, \mathrm{P}<0.01)$.

\section{Discussion}

The majority of studies show that the renin-angiotensin system (RAS) is activated by AF, and simultaneously, as a major effecter molecule of the RAS in circulating and certain tissues (11), AngII levels increase and eventually induce atrial fibrosis (3). The present study identified that AngII levels in the ISO and Rapa groups in myocardial tissue were significantly higher than the control group after seven days of subcutaneous multiple high-dose continuous injection of ISO in rats, which implies that following ISO injection, RAS was activated and caused increased expression levels of AngII in myocardial tissue. According to the morphological observation, no atrial fibrosis was identified in the control group, while the ISO 
group had significant myocardial interstitial fibrosis, which indicates that high expression of AngII in myocardial tissues may be involved in atrial fibrosis formation, and this has been confirmed by a previous study (12).

The level of HIF-1 $\alpha$ expression, as a tissue hypoxia index product, will increase during tissue hypoxia. Hypoxia has been linked to fibrosis (13), including in the liver $(14,15)$, lung (16) and kidney (17). Although studies have shown that the increase in $H I F-1 \alpha$ gene expression in the myocardium may be involved in its structural changes, including atrial fibrosis $(18,19)$, no study has been conducted for the elevated AngII-induced myocardial fibrosis. The present study, from the aspects of pathology and protein and mRNA expression levels, found that the expression of HIF-1 $\alpha$ in the ISO group was significantly higher than that in the control group, while when administering the HIF-1 $\alpha$ inhibitor rapamycin intervention in the Rapa group, HIF-1 $\alpha$ expression decreased significantly, and was positively correlated with myocardial fibrosis degree (CVF), which proves that AngII is involved in atrial fibrosis by regulating the expression of HIF-1 $\alpha$.

TGF- $\beta_{1}$, as one of the AngII downstream factors (20), is associated with myocardial fibrosis occurrence (21). The present study also provides evidence for this. HIF-1 $\alpha$ has a close association with TGF- $\beta_{1}$ and can regulate its expression $(22,23)$. The present study shows that in the ISO group, $T G F-\beta_{1}$ mRNA expression levels were much higher than those in the control group, and its expression in the Rapa group was significantly reduced. The expression of HIF-1 $\alpha$ and the extent of myocardial fibrosis was positively correlated, which implies that HIF-1 $\alpha$ can facilitate the expression level of TGF- $\beta_{1}$ and thus induce atrial fibrosis.

In patients with $\mathrm{AF}$, it has been reported that the atrial HIF-1 $\alpha$ level rises with the increasing expression level of MMP-9 (24). MMP-9, as an important protease in the MMP family, is associated with myocardial matrix remodeling (25), and its increasing activity can result in acute myocardial fibrosis $(26,27)$. The present study shows that in the ISO group, $H I F-1 \alpha$ and MMP-9 mRNA expression levels were significantly increased. They were positively correlated between themselves and also positively correlated with atrial fibrosis. Furthermore, HIF-1 $\alpha$ can be involved in myocardial fibrosis formation by regulating the MMP-9 expression level. TGF- $\beta_{1}$ is closely associated with MMP-9 and can regulate its gene level expression (28) and thus induce its synthesis (29). In the present study, it was also observed that in the ISO group, the MMP-9 mRNA expression level was markedly higher than in the control group, while in the Rapa group this was significantly decreased. The expression level of TGF- $\beta_{1}$ was also found to be positively associated with the degree of myocardial fibrosis. These results imply that TGF- $\beta_{1}$ expression levels increase and cause the high expression of MMP-9, and thus aggravates myocardial fibrosis, which could be a possible mechanism in the AngII-induced atrial fibrosis model.

In conclusion, the present study shows that in the ISO-induced atrial fibrosis model, AngII, HIF- $1 \alpha$, TGF- $\beta_{1}$ and MMP-9 were all highly expressed. By inhibiting the expression of HIF- $1 \alpha$, the expression levels of TGF- $\beta_{1}$ and MMP-9 decreased accordingly, and the extent of myocardial fibrosis was also reduced. Considering the association among these factors, we can infer that, during the atrial fibrosis formation, HIF- $1 \alpha$ promotes the expression of TGF- $\beta_{1}$ and MMP-9 protein. A possible signal transduction pathway among HIF-1 $\alpha$, TGF- $\beta_{1}$ and MMP-9 may exist, which could contribute significantly to the further study of the pathogenesis of AF and a new direction of drug research and development in AF therapy.

\section{References}

1. Meiltz A, Zimmermann M, Urban P, Bloch A; Association of Cardiologists of the Canton of Geneva: Atrial fibrillation management by practice cardiologists: a prospective survey on the adherence to guidelines in the real world. Europace 10: 674-680, 2008

2. Benjamin EJ, Levy D, Vaziri SM, D'Agostino RB, Belanger AJ and Wolf PA: Independent risk factors for atrial fibrillation in a population-based cohort. The Framingham Heart Study. JAMA 271: 840-844, 1994.

3. Pan CH, Lin JL, Lai LP, Chen CL, Stephen Huang SK and Lin CS: Downregulation of angiotensin converting enzyme II is associated with pacing-induced sustained atrial fibrillation. FEBS Lett 581: 526-534, 2007.

4. Frustaci A, Chimenti C, Bellocci F, Morgante E, Russo MA and Maseri A: Histological substrate of atrial biopsies in patients with lone atrial fibrillation. Circulation 96: 1180-1184, 1997.

5. Everett TH IV, Li H, Mangrum JM, et al: Electrical, morphological, and ultrastructural remodeling and reverse remodeling in a canine model of chronic atrial fibrillation. Circulation 102: 1454-1460, 2000.

6. LiD, Shinagawa K, Pang L, et al: Effects of angiotensin-converting enzyme inhibition on the development of the atrial fibrillation substrate in dogs with ventricular tachypacing-induced congestive heart failure. Circulation 104: 2608-2614, 2001.

7. Wang Z, Tang L, Zhu Q, et al: Hypoxia-inducible factor- $1 \alpha$ contributes to the profibrotic action of angiotensin II in renal medullary interstitial cells. Kidney Int 79: 300-310, 2011.

8. Zhang YG, Li YG, Liu BG, et al: Urotensin II accelerates cardiac fibrosis and hypertrophy of rats induced by isoproterenol. Acta Pharmacol Sin 28: 36-43, 2007.

9. Hudson CC, Liu M, Chiang GG, et al: Regulation of hypoxia-inducible factor lalpha expression and function by the mammalian target of rapamycin. Mol Cell Biol 22: 7004-7014, 2002.

10. Dong Y, Huang YF, Wang LQ and Chen B: Experimental study on the effects of rapamycin in prevention of rat corneal allograft rejection. Zhonghua Yan Ke Za Zhi 41: 930-935, 2005 (In Chinese).

11. Matsushita K, Wu Y, Okamoto Y, Pratt RE and Dzau VJ: Local renin angiotensin expression regulates human mesenchymal stem cell differentiation to adipocytes. Hypertension 48: 1095-1102, 2006.

12. Zhang WZ, Wang ZG, Chen YQ, et al: Effects of valsartan and U0126 on atrial fibrosis and connexin40 remodeling in rats. Zhonghua Xin Xue Guan Bing Za Zhi 39: 1129-1134, 2011 (In Chinese).

13. Fine LG and Norman JT: Chronic hypoxia as a mechanism of progression of chronic kidney diseases: from hypothesis to novel therapeutics. Kidney Int 74: 867-872, 2008.

14. Copple BL, Kaska S and Wentling C: Hypoxia-inducible factor activation in myeloid cells contributes to the development of liver fibrosis in cholestatic mice. J Pharmacol Exp Ther 341: 307-316, 2012.

15. Rosmorduc O and Housset C: Hypoxia: a link between fibrogenesis, angiogenesis, and carcinogenesis in liver disease. Semin Liver Dis 30: 258-270, 2010.

16. Ueno M, Maeno T, Nomura M, et al: Hypoxia-inducible factor-1 $\alpha$ mediates TGF- $\beta$-induced PAI- 1 production in alveolar macrophages in pulmonary fibrosis. Am J Physiol Lung Cell Mol Physiol 300: L740-L752, 2011.

17. Haase VH: Hypoxia-inducible factor signaling in the development of kidney fibrosis. Fibrogenesis Tissue Repair 5 Suppl 1: S16, 2012.

18. Thijssen VL, van der Velden HM, van Ankeren EP, et al: Analysis of altered gene expression during sustained atrial fibrillation in the goat. Cardiovasc Res 54: 427-437, 2002.

19. Gramley F, Lorenzen J, Jedamzik B, et al: Atrial fibrillation is associated with cardiac hypoxia. Cardiovasc Pathol 19: 102-111, 2010. 
20. Rosenkranz S, Flesch M, Amann K, et al: Alterations of beta-adrenergic signaling and cardiac hypertrophy in transgenic mice overexpressing TGF-beta(1). Am J Physiol Heart Circ Physiol 283: H1253-H1262, 2002.

21. Lim JY, Park SJ, Hwang HY, et al: TGF-beta1 induces cardiac hypertrophic responses via PKC-dependent ATF-2 activation. J Mol Cell Cardiol 39: 627-636, 2005.

22. Mihout F, Shweke N, Bigé N, et al: Asymmetric dimethylarginine (ADMA) induces chronic kidney disease through a mechanism involving collagen and TGF- $\beta 1$ synthesis. J Pathol 223: 37-45, 2011.

23. Higgins DF, Kimura K, Bernhardt WM, et al: Hypoxia promotes fibrogenesis in vivo via HIF-1 stimulation of epithelial-to-mesenchymal transition. J Clin Invest 117: 3810-3820, 2007.

24. Ogi H, Nakano Y, Niida S, et al: Is structural remodeling of fibrillated atria the consequence of tissue hypoxia? Circ J 74: 1815-1821, 2010.

25. IsobeK,KubaK,Maejima Y,etal: Inhibition of endostatin/collagen XVIII deteriorates left ventricular remodeling and heart failure in rat myocardial infarction model. Circ J 74: 109-119, 2010.
26. Xu J, Cui G, Esmailian F, et al: Atrial extracellular matrix remodeling and the maintenance of atrial fibrillation. Circulation 109: 363-368, 2004.

27. Nakano Y, Niida S, Dote K, et al: Matrix metalloproteinase-9 contributes to human atrial remodeling during atrial fibrillation. J Am Coll Cardiol 43: 818-825, 2004.

28. Santibañez JF, Guerrero J, Quintanilla M, Fabra A and Martínez J: Transforming growth factor-beta 1 modulates matrix metalloproteinase-9 production through the Ras/MAPK signaling pathway in transformed keratinocytes. Biochem Biophys Res Commun 296: 267-273, 2002.

29. Zhang BB, Cai WM, Weng HL, et al: Diagnostic value of platelet derived growth factor-BB, transforming growth factor-beta1, matrix metalloproteinase-1, and tissue inhibitor of matrix metalloproteinase- 1 in serum and peripheral blood mononuclear cells for hepatic fibrosis. World J Gastroenterol 9: 2490-2496, 2003. 